\title{
Cerebellar-dependent delay eyeblink conditioning in adolescents with Specific Language Impairment
}

\author{
Adam B. Steinmetz • Mabel L. Rice
}

Received: 2 August 2009 / Accepted: 28 July 2010 / Published online: 10 August 2010

(C) Springer Science+Business Media, LLC 2010

\begin{abstract}
Cerebellar impairments have been hypothesized as part of the pathogenesis of Specific Language Impairment (SLI), although direct evidence of cerebellar involvement is sparse. Eyeblink Conditioning (EBC) is a learning task with well documented cerebellar pathways. This is the first study of EBC in affected adolescents and controls. 16 adolescent controls, 15 adolescents with SLI, and 12 adult controls participated in a delay EBC task. Affected children had low general language performance, grammatical deficits but no speech impairments. The affected group did not differ from the control adolescent or control adult group, showing intact cerebellar functioning on the EBC task. This study did not support cerebellar impairment at the level of basic learning pathways as part of the pathogenesis of SLI. Outcomes do not rule out cerebellar influences on speech impairment, or possible other forms of cerebellar functioning as contributing to SLI.
\end{abstract}

Keywords Specific Language Impairment · Cerebellum . Eyeblink conditioning · Conditioned learning .

Adolescence

\author{
A. B. Steinmetz \\ Department of Psychology, University of Iowa, \\ E11 Seashore Hall, \\ Iowa City, IA 52242, USA \\ M. L. Rice ( $\square)$ \\ Department of Speech-Language-Hearing: Sciences \& Disorders, \\ University of Kansas, \\ 1000 Sunnyside Ave., \\ Lawrence, KS 66045, USA \\ e-mail: mabel@ku.edu
}

Specific Language Impairment (SLI) is diagnosed in children with language impairments without an obvious cause (i.e. hearing loss, cognitive or physical impairment). The overall prevalence rate for SLI in epidemiologically ascertained samples of healthy children is $7.4 \%$ ( $8 \%$ for boys and 6\% for girls) (Tomblin et al. 1997). Grammatical deficits are widely recognized as persistent clinical markers of SLI (Rice et al. 2009a, 1995; Rice and Wexler 1996; Rice 2000; Tager-Flusberg and Cooper 1999). Deficits in motor speech production are relatively independent of SLI, appearing in $5-8 \%$ of young affected children although clinically ascertained samples can have a higher percentage (Shriberg et al. 1999). Group comparisons of affected and age-matched control children reveal subtle differences in performance on motor tasks (Bishop 2002; Zubrick et al. 2007; Zelaznik and Goffman 2010), longer latencies on perceptual/cognitive tasks (Leonard et al. 2007), and possible attentional deficits (Lum et al. 2007).

Children with SLI show overlap with other developmental disabilities. Two are of interest here. One is reading impairment. Studies yield an estimate of $50 \%$ of young children with SLI who subsequently develop reading impairments (Catts 2004). The language symptoms of SLI are also evident in children with Autism Spectrum Disorders (ASD). Recent studies report similar grammar phenotypes of children with ASD and children with SLI (Roberts et al. 2004; Kjelgaard and TagerFlusberg 2001). A recent study of children with a history of SLI found the prevalence of ASD in this sample higher than that of the general population (Conti-Ramsden et al. 2006). 
The pathogenesis of SLI is unknown. The biological underpinnings are attracting new attention as candidate genes are discovered for SLI, such as KIAA 0319 for several language and reading phenotypes (Rice et al. 2009b) and for ASD, such as CNTNAP2, based on a phenotype of late language emergence during the toddler period (Alarcón et al. 2008). In turn, CNTNAP2 polymorphisms in children with SLI showed quantitative associations with language-related behavioral phenotypes in a sample of children with SLI (Vernes et al. 2008). Further, the region of association coincides with a region reported for language delays in children with autism (Alarcón et al. 2008). Both KIAA 0319 and CNTNAP2 are expressed in the developing human cortex, pointing toward pathways in brain functioning as important elements in the pathogenesis of language impairments. On the other hand, a recent investigation of the first degree relatives of children with SLI and children with ASD did not find support for similar genetic loading for language (Lindgren et al. 2009).

Recent theories posit a role for the cerebellum in the pathogenesis of SLI (Hill 2001), with a particular emphasis on subtle impairments in motor output and attentional processes as concomitant indicators of possible cerebellar involvement. The cerebellum has been thought to be solely a part of motor control, but more recently it has been implicated in cognition (Rapoport et al. 2000), selective attention (Akshoomoff and Courchesne 1992; Yamaguchi et al. 1998), and language skills (Leiner et al. 1991), including morphological deficits in adults with cerebellar injuries (Justus 2004). The complex interconnections of the cerebellum with frontal cortical processes warrants more investigation of the cerebellum's effects on children's development (Diamond 2000). A cerebellar deficit hypothesis, based in the underlying neural substrate, has been proposed for developmental dyslexia (Nicolson et al. 2001), in which cerebellar impairment is predicted to affect several pathways of behavioral development. One direct manifestation of cerebellar impairment is predicted to be impaired articulatory skill which in turn leads to reading impairments. Another hypothesized pathway is motor skill impairment and/or balance impairment that leads to writing impairments, and a third pathway predicts that problems with automatising skill and knowledge lead to reading and spelling problems.

The current theoretical models of possible impaired cerebellar functions for SLI or dyslexia are rather sketchy in nature, invoking a relatively wide range of related symptoms and an unspecified locus of cerebellar dysfunction (see a recent paper by Nicolson and Fawcett (Nicolson and Fawcett 2007) for an over-arching conceptual framework and discussion of some of the issues in differentiating the functions of components of cortical and cerebellar functioning). In contrast, there is evidence of a precise dysfunction in cerebellar processing in children with autism (Sears et al. 1994), utilizing classical eyeblink conditioning $(E B C)$ as an index of basic learning pathways linked to a motor response. This experimental task has been widely used to probe cerebellar function in humans and nonhuman mammals.

In the most common form of the $\mathrm{EBC}$ procedure, termed single-cue delay EBC, a conditioned stimulus (CS; e.g., $400 \mathrm{~ms}$ tone) is paired with a co-terminating unconditioned stimulus (US; e.g., $50 \mathrm{~ms}$ corneal airpuff). Repeated CSUS pairings elicit the development of an adaptive conditioned blink response (CR), which occurs in healthy individuals just prior to the US. The delay EBC procedure appears to be the purest assay of the functional integrity of the cerebellum, with the circuitry and synaptic mechanisms being well studied and identified in nonhumans (Kim and Thompson 1997; Steinmetz 2000; Christian and Thompson 2003) compared to other forms of EBC, such as trace conditioning (Christian and Thompson 2003). In addition, the cerebellar networks mediating this form of associative learning appear to be conserved across mammals (rat: (Rogers et al. 2001); human: (Gerwig et al. 2007)). The single-cue delay procedure has been used to examine developmental and clinical conditions associated with cerebellar abnormalities and motor disruptions, such as aging (e.g., (WoodruffPak and Thompson 1988; Woodruff-Pak et al. 1999)), autism(Sears et al. 1994), schizophrenia (e.g., (Brown et al. 2005)) and drug abuse (Skosnik et al. 2008). In their study of children with autism ages 7-22 years, Sears et al. (1994) report that affected children differed from controls with faster rates of conditioning at a young age and maintained that rate as age increased whereas control subjects showed increasingly fast rates of conditioning with age. In addition, the autism group showed a more rapid and significant decline in the amplitudes of conditioned responses during an extinction phase. The topography of the conditioned responses of the autism group differed from the control group. Overall, the patterns of motor learning associated with cerebellar functioning differentiated the children with autism from the control group.

Somewhat different methods for evaluating the EBC were used in a study of 13 adolescents and young adults with dyslexia, ages 13-24 years, and 13 same-age controls (Nicolson et al. 2002). The conclusion was that the dyslexic group performed poorly relative to the control group, showing either no conditioning or poor timing of CRs and/or abnormally low orienting responses. The general conclusion of the study is that the outcomes provide evidence of fundamental differences in the way people with dyslexia learn. 
In sum, the underlying cerebellar pathways involved in the EBC have been mapped in great detail, with converging evidence from studies of humans and other mammals. Research during the past 25 years strongly supports the role of the cerebellar cortex and deep nuclei in the acquisition and timing of the $\mathrm{CR}$ during delay $\mathrm{EBC}$. In relation to learning and behavioral occurrence of the CR, lesion, neural unit recording, and reversible inactivation studies have provided compelling evidence that the memory trace for delay EBC resides in regions of the cerebellar deep nuclei (anterior lateral interpositus nucleus ipsilateral to the trained eye; for review see (Christian and Thompson 2003; Steinmetz 2000)). While it is difficult to assess the effect of interpositus lesions on EBC performance in humans (since in many clinical cases, the cerebellar cortex is also affected; (McGlinchey-Berroth et al. 1995; Topka et al. 1993; Daum et al. 1993; Solomon et al. 1989; Lye et al. 1988), it has been suggested that most of the patient studies demonstrating delay EBC deficits after cerebellar insult include damage to the deep nuclei (Schugens and Daum 1999).

While evidence clearly suggests that the cerebellar deep nuclei are involved in the acquisition of the CR, several studies have also identified a possible role of the cerebellar cortex in the timing and gain of CRs (Lavond and Steinmetz 1989; McCormick and Thompson 1984; Logan 1991; Perrett et al. 1993). In humans, the involvement of the cerebellar cortex in modulating the $\mathrm{CR}$ has been supported using positron emission tomography (Blaxton et al. 1996; Logan and Grafton 1995), functional MRI (Ramnani et al. 2000; Dimitrova et al. 2002) and clinical samples with cerebellar insults (Daum et al. 1993; Woodruff-Pak et al. 1996). More recently, it has been demonstrated that in cerebellar patients, damage to the anterior lobe was significantly correlated with timing deficits during delay EBC as assessed via MRI (Gerwig et al. 2005). One possible conclusion that can be drawn from these studies is that a dynamic interaction between the cerebellar cortex and deep nuclei underlies both the acquisition and the accurate timing of the $\mathrm{CR}$, with the cortex potentially playing a crucial role in modulating the timing of the response.

Studies of children with autism document a pattern of EBC different in the affected versus control children. Although the language abilities of the children in the previous study (Sears et al. 1994) were not described in the research report, the outcomes suggest that the EBC is a candidate for a cerebellar pathway that could be impaired in children with language impairments, to the extent that children with SLI and children with autism share underlying brain pathways for language impairments. Further, previous studies of children and young adults with dyslexia (Nicolson et al. 2002) and dyslexia subsequent to prenatal alcohol exposure (Coffin et al. 2005) report impaired EBC for the affected group. The study reported here addresses the need for a study using the delay eyeblink conditioning paradigm to test the functional integrity of the cerebellum in subjects diagnosed with SLI. This paradigm consisted of both an acquisition and extinction period to examine the learning and un-learning of the response, following important precedents in the literature that document the full pattern of motor response learning. The hypotheses were that the children with SLI would exhibit fewer CRs than controls (assuming subtle motor learning impairments) and the timing of the CR should also be impaired with worse timing, as in the previous study of children with autism. The participants in this study did not have speech impairments, providing an opportunity to investigate whether the hypothesis of cerebellar impairment is supported in a sample of children with language impairments without speech impairments.

\section{Methods}

\section{Participants}

There were a total of 43 participants, four groups of children $(N=31)$ and one adult control group $(N=12)$. The children were grouped as follows: 6 young controls (age range $=9.1-11.11$, mean $=10.1$, s.d. $=1.1), 10$ older controls (age range $=17.3-19 ; 9 ;$ mean age $=18.30$, s.d. $=0 ; 11), 5$ young SLI (age range $=9.7-12.8$; mean age $=11 ; 7$, s.d., $=1 ; 5$ ), 10 older SLI (age range $=14.6-19.9$; mean $=17 ; 0$, s.d. $=2.1$ ) Collapsed across ages, there were 11 males in the control groups and 12 males in the SLI group. All children were participants in an ongoing longitudinal study documenting long-term language growth patterns. Children in the SLI group entered the longitudinal study as affected children, initially identified by clinical speech/language pathologists. The children were experimentally screened for speech impairments. The criterion was defined by a passing score on a probe screening for articulation competency with consistent use of final $-t,-d$, and $-z$ (Rice and Wexler 2001) and only minor mispronunciations, such as distortions of $/ \mathrm{r} /, / 1 /$ and blends, on the Goldman-Fristoe Test of Articulation-2 (GFTA-2) (Goldman and Fristoe 1986). The hearing criterion at entry was passage of a screening at $25 \mathrm{~dB} \mathrm{HL}$ at 1,000, 2,000, and 4,000 Hz. Children with diagnoses of autism or autism spectrum disorders were excluded, as were children with nonverbal intelligence levels below 85 on the Columbia Mental Maturity Scales (CMMS) (Burgemeister et al. 1972)or the Wechsler Intelligence scale for Children (WISC): Nonverbal Scale (Wechsler 1991). At entry all children in the SLI group performed one standard deviation or more below the mean on an ageappropriate omnibus standardized language assessment. Further, children's reading ability was assessed on the 
Woodcock Reading Mastery Tests-Revised (Woodcock 1987), Word Identification subtest. As summarized in Table 1, the archival data files show that at initial testing the group of children with SLI were equivalent to the control children in total number of articulation errors but at significantly lower levels of performance on omnibus language assessment, vocabulary, and word identification levels. The ages of the children at initial testing for each measure are provided in Table 1. They were somewhat older at initial testing for reading, given the ages at which reading instruction started in the schools. Also summarized in Table 1 are assessments concurrent with the eyeblink data collection. As at the initial testing, the two groups do not differ on total number of articulation errors. The concurrent assessments confirm the SLI group continues to perform lower on omnibus language assessment, vocabulary (although the SLI group's mean vocabulary performance was within normal range), and word identification. Further, persistent grammatical deficits were apparent on an experimental grammaticality judgment task administered at the time of the experiment. Control children were more likely to detect the ungrammaticality of sentences such as Where the dog is playing?, with A' mean=.97 where 1.00 is the ideal performance for the adult grammar, compared to $\mathrm{A}^{\prime}=.82$ for the SLI group $(t(15.64$, adjusted for unequal variances $)=$ 3.466, $p=.003$ (two-tailed), $d=2.10$. At the same time, as expected, the two groups did not differ in judgments of grammatical sentences such as Where is the dog playing?/Is the dog playing? Thus, the SLI group's language performance was consistently low over time, although the mean level of speech production did not differentiate the groups at outset in the larger parent study when they were much younger, nor at the time of the eyeblink data collection. Also, as expected, the SLI group's mean performance on a word identification task documents a persistent risk for reading impairment. Because the study was focused on detailed documentation of speech, language, and reading acquisition, no measures of motor performance were included in the longitudinal protocol. In addition, 12 adult controls (mean age $=25.67$, s.d. 2.05) were recruited via fliers from the local community. Prior to the experimental task, all participants completed an audiology pure tone screening at $500 \mathrm{~Hz}$, $1,000 \mathrm{~Hz}, 2,000 \mathrm{~Hz}$, and $4,000 \mathrm{~Hz}$; criterion for participation was defined as passing at $25-30 \mathrm{~dB}$ in at least one ear for each of the frequencies.

EBC stimuli and procedure

Participants completed a 133-trial delay EBC paradigm similar to (Sears et al. 1994) and (Brown et al. 2005). Initially, eight US alone trials were presented with an intertrial interval (ITI) of $15 \mathrm{~s}$. Without interruption, the

Table 1 Speech, language, and reading mean scores and standard deviations per group

\begin{tabular}{|c|c|c|c|c|c|c|c|}
\hline & \multicolumn{2}{|l|}{ SLI } & \multicolumn{2}{|l|}{ Control } & \multirow[t]{2}{*}{$p$ value } & \multirow[t]{2}{*}{$\mathrm{df}$} & \multirow{2}{*}{$\begin{array}{l}\text { Cohen's d } \\
\text { effect size }\end{array}$} \\
\hline & Mean & SD & Mean & SD & & & \\
\hline \multicolumn{8}{|l|}{ Initial testing } \\
\hline GFTA total errors & 10.20 & 7.61 & 6.44 & 6.86 & 0.158 & 29 & 0.55 \\
\hline GFTA age & 6,11 & 1,6 & 4,7 & 1,7 & & & \\
\hline Omnibus language $^{\mathrm{a}}$ & 73.53 & 7.76 & 119.69 & 17.27 & 0.000 & 29 & 3.81 \\
\hline Omnibus age & 6,9 & 1,7 & 4,7 & 1,7 & & & \\
\hline Vocabulary $^{\mathrm{b}}$ & 83.87 & 15.47 & 106.44 & 12.22 & 0.000 & 29 & 1.85 \\
\hline Vocabulary age & 6,9 & 1,7 & 4,7 & 1,7 & & & \\
\hline Word ID & 83.93 & 11.41 & 104.94 & 12.30 & 0.000 & 29 & 1.71 \\
\hline Word ID age & 7,7 & 1,0 & 5,11 & 1,0 & & & \\
\hline \multicolumn{8}{|l|}{ Concurrent testing } \\
\hline GFTA total errors & 2.07 & 1.71 & 2.06 & 2.65 & 0.996 & 29 & 0.00 \\
\hline Omnibus language ${ }^{c}$ & 83.33 & 15.38 & 107.50 & 10.33 & 0.000 & 29 & 2.34 \\
\hline Grammar judgements & 0.82 & 0.16 & 0.97 & 0.04 & 0.001 & 29 & 3.75 \\
\hline Vocabulary $^{\mathrm{d}}$ & 97.67 & 9.48 & 111.69 & 8.28 & 0.000 & 29 & 1.69 \\
\hline Word ID & 81.47 & 13.63 & 103.81 & 11.79 & 0.000 & 29 & 1.89 \\
\hline
\end{tabular}

${ }^{a}$ Omnibus language score is the summative standard score for Test of Early Language Development (TELD-2 or TELD-3), Test of Language DevelopmentPrimary 2nd Edition (TOLD P:2), or Clinical Evaluation of Language Fundamentals 3rd edition (CELF-3)

${ }^{\mathrm{b}}$ Vocabulary score is the summative standard score for the Peabody Picture Vocabulary Test-R (Revised) or Peabody Picture Vocabulary Test-3

${ }^{\mathrm{c}}$ Omnibus language score is the summative standard score for Clinical Evaluation of Language Fundamentals 3rd Edition (CELF-3)

${ }^{\mathrm{d}}$ Vocabulary score is the summative standard score for the Peabody Picture Vocabulary Test- 3 
acquisition phase immediately followed and consisted of 10 trial blocks (mean ITI $=15 \mathrm{~s}$, range $=10-20 \mathrm{~s}$ ), each containing $10 \mathrm{CS}-\mathrm{US}$ paired trials. Paired CS-US trials consisted of a $400 \mathrm{~ms}, 1,000 \mathrm{~Hz}$ tone ( $80 \mathrm{~dB}$ SPL) with a co-terminating $50 \mathrm{~ms}$ air puff. The procedure concluded with an extinction phase consisting of $25 \mathrm{CS}$ alone trials presented across blocks (mean ITI $=15 \mathrm{~s}$; range $=10-20 \mathrm{~s}$ ). To maintain attention throughout the procedure, participants watched the movie Milo and Otis with no sound. Subjects were observed through a closed circuit monitor in order to observe alertness. The experiment was briefly suspended if signs of fatigue were observed so that the examiner could interact with the participant.

Eyeblinks were recorded using pairs of electromyographic (EMG) electrodes (8 mm Ag/AgCl; Model TD-23; MedAssociated, St Albans, VT). Bipolar recording electrodes, with conductive gel applied, were placed on the orbicularis palpebrarum muscle below the left eye. A ground electrode was placed on the forehead. All electrode impedances were maintained below $10 \mathrm{k} \Omega$. The US consisted of a 10 p.s.i. (50 ms duration) puff of medical-grade air presented to the left eye with tubing affixed to eye-glass rims and positioned $1 \mathrm{~cm}$ away from the inner canthus of the eye. Foam ear inserts were used for presentation of the CS tones (E-A-RLINK, Aearo Company Auditory Systems, Indianapolis, IN). EMG data were continuously recorded at $1.0 \mathrm{kHz}$ with a Sensorium EPA-6 bioamplifier (highpass filter $=1 \mathrm{~Hz}$, $12 \mathrm{~dB} /$ octave; lowpass filter $=300 \mathrm{~Hz}$, eighth order elliptic; gain $=5,000$ ) and acquired using Brainvison software (v. x.x, Richardson, Texas).

\section{EBC data processing}

Individual trials were epoched from the continuous EMG data file (using Brainvision Edit software), and filtered (10 Hz high pass filter; $6 \mathrm{~dB} /$ octave) before being rectified and smoothed using a 41-point Gaussian weighted moving average. Data were then entered into the Data-Munch software program for further analysis (King and Datamunch 1999). For each subject, responses were recorded as blinks if the amplitude exceeded five standard deviations above the baseline (baseline window for each trial $=125 \mathrm{~ms}$ before CS presentation). CRs were recorded if the blink occurred between 100 and $350 \mathrm{~ms}$ after CS onset (corresponding to a period beginning $250 \mathrm{~ms}$ before US onset). The onset latency was calculated as the point in time where the conditioned response exceeded 5.0 standard deviations from the baseline. The peak latency is the time point for the maximal value for that conditioned response. Trials in which spontaneous blinks occur within a window from $75 \mathrm{~ms}$ before CS presentation to $25 \mathrm{~ms}$ following CS onset were labeled bad trials and excluded from further analysis.
Statistical analysis

The primary dependent measures for the eyeblink procedure were percentage of CRs, CR onset latency, CR peak latency, CR amplitude, and UR amplitude. For each of these variables, the majority of the analyses were conducted with a mixed model ANOVA with two levels of a group factor (i.e. control, SLI) and 10 levels of a within-subjects block factor. The group factor was a between-subjects factor when within-session effects were examined whereas it was a within-subjects factor for analyses across session. An analogous analytical model was used to examine extinction data, except that the block factor had only 5 levels. For the primary dependent variables, effect sizes are reported by partial $\eta^{2}$, where small effect sizes are less than 0.06 , moderate effect sizes range from 0.06 to 0.14 , and large effect sizes are greater than 0.14 (Cohen 1973). All statistical tests used an alpha level of $p<0.05$ to determine significance (two-tailed). If Mauchly's Test of Sphericity was significant, Greenhouse-Geisser was used to determine significance. All tests were performed using the software package SPSS 14.0.

\section{Results}

Age effects

Young controls (age 9-12), older controls (Catts 2004; Roberts et al. 2004; Kjelgaard and Tager-Flusberg 2001; Conti-Ramsden et al. 2006; Rice et al. 2009b; Alarcón et al. 2008; Vernes et al. 2008) and adult controls (Akshoomoff and Courchesne 1992; Yamaguchi et al. 1998; Leiner et al. 1991; Justus 2004; Diamond 2000; Nicolson et al. 2001; Nicolson and Fawcett 2007; Sears et al. 1994) were compared in order to examine possible learning effects of age. A repeated measures ANOVA for percentage of CRs during the acquisition phase revealed a main effect of block, $F(9,17)=3.12 p=0.02$; partial $\eta^{2}=.62$, but no significant main effect of age (partial $\eta^{2}=.02$ ) and no block $\times$ age interaction (partial $\eta^{2}=.40$ ). The extinction phase revealed a main effect of block, $F(4,22)=8.81 p<$ 0.001; partial $\eta^{2}=.62$, but no significant main effect of age (partial $\eta^{2}=.04$ ) and no block $\times$ age interaction (partial $\eta^{2}=.16$ ). For CR peak latencies there was a main effect of block, $F(9,17)=4.42 p=0.001$; partial $\eta^{2}=.15$, but no main effect of age (partial $\eta^{2}=.11$ ) or block $\times$ age interaction (partial $\eta^{2}=.06$ ). No statistical effects were revealed with regards to $\mathrm{CR}$ onset, $\mathrm{CR}$ amplitude, UR amplitude, or during the extinction phase. In summary, the conditioned responses were learned at a similar rate and did not differ between the age groups; timing and amplitudes also did not differ between the groups. For further analysis, the young 
controls and the older controls were combined to form one control group.

In order to examine possible age effects on learning, younger SLI participants (age 9-12) were compared to older SLI participants (Catts 2004; Roberts et al. 2004; Kjelgaard and Tager-Flusberg 2001; Conti-Ramsden et al. 2006; Rice et al. 2009b; Alarcón et al. 2008; Vernes et al. 2008). A repeated measures ANOVA for percentage of CRs during acquisition revealed a main effect of block, $F(5,9)=$ $3.12 p=0.05$; partial $\eta^{2}=.62$, but no significant main effect of age (partial $\left.\eta^{2}=.01\right)$ and no block $\times$ age interaction (partial $\eta^{2}=.15$ ). The extinction phase revealed a main effect of block, $F(4,10)=3.09 p=0.049$; partial $\eta^{2}=.61$, but no significant main effect of age (partial $\eta^{2}=.10$ ) and no block $\times$ age interaction (partial $\eta^{2}=.20$ ). For $C R$ peak latencies there was a main effect of block, $F(5,9)=14.35$ $p=0.005$; partial $\eta^{2}=.96$, but no main effect of age (partial $\eta^{2}=.80$ ) or block $\times$ age interaction (partial $\eta^{2}=.56$ ). No statistical effects were revealed with regards to CR onset, CR amplitude, UR amplitude, or during the extinction phase. In summary, the conditioned responses were learned at a similar rate and did not differ between the age groups; timing and amplitudes also did not differ between the groups. For further analysis, the younger SLI participants and the older SLI participants were combined to form one SLI group.

\section{SLI vs controls}

Percent CRs, CR onset latencies, CR peak latencies, CR amplitudes, and UR amplitudes during each 10-trial block (plus extinction) were calculated for each group. For $\%$ CRs (Fig. 1), a repeated measures ANOVA revealed a main effect of block, $F(9,21)=5.70 p<0.001$; partial

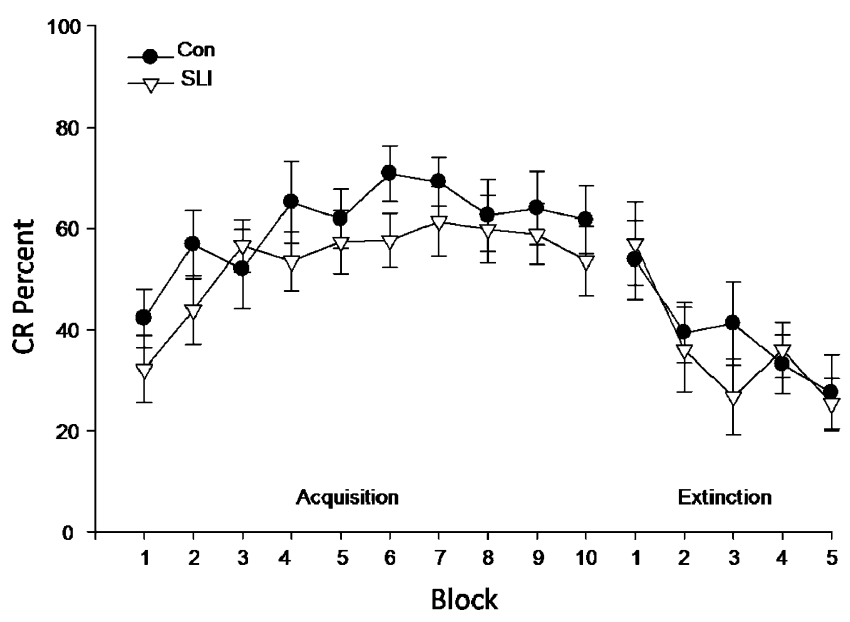

Fig. 1 Percent conditioned responses for the control and SLI participants. No significant differences were observed between the two groups $\eta^{2}=.71$. However, there was no main effect of group (partial $\eta^{2}=.03$ ) and no block $\times$ group interaction (partial $\eta^{2}=.53$ ). For CR peak latency, there was also a main effect of block, $F(9,21)=5.16 p<0.001$; partial $\eta^{2}=.15$, but no main effect of group (partial $\eta^{2}=.01$ ) and no block $\times$ group interaction (partial $\eta^{2}=.02$ ). There were no significant effects with regards to CR onset, CR amplitude, and UR amplitude. The extinction phase revealed a main effect of block, $F(4,26)=7.97 p<0.001$; partial $\eta^{2}=.22$, but no significant main effect of group (partial $\eta^{2}=.01$ ) or block $\times$ group interaction (partial $\eta^{2}=.03$ ). Thus, the two groups learned the response at similar rates and the timings of the responses did not differ. No differences regarding PPVT scores were observed when the SLI participants were compared to the control participants $(p=.187)$. There were no significant correlations found when the PPVT was correlated to mean $\mathrm{CR} \%$ (Fig. 2), $\mathrm{CR}$ peak latencies, $\mathrm{CR}$ onset latencies, CR amplitudes, and UR amplitudes. There were also no significant values when each of the conditioning variables were split to first half (blocks 15 ) and second half (blocks 6-10). In order to examine variance between the groups, a one way ANOVA was used to compare the variance in percent CRs between the SLI and control groups. The ANOVA revealed no significant difference between the groups $F(1,29)=0.96 p=0.34$; partial $\eta^{2}=.23$. This finding suggests that the two groups' variance was not significantly different.

\section{Discussion}

Overall, this is the first study to employ delay eyeblink conditioning, a cerebellar-dependent task, with children with SLI, in order to evaluate hypotheses generated from models of cerebellar impairment contributing to speech,

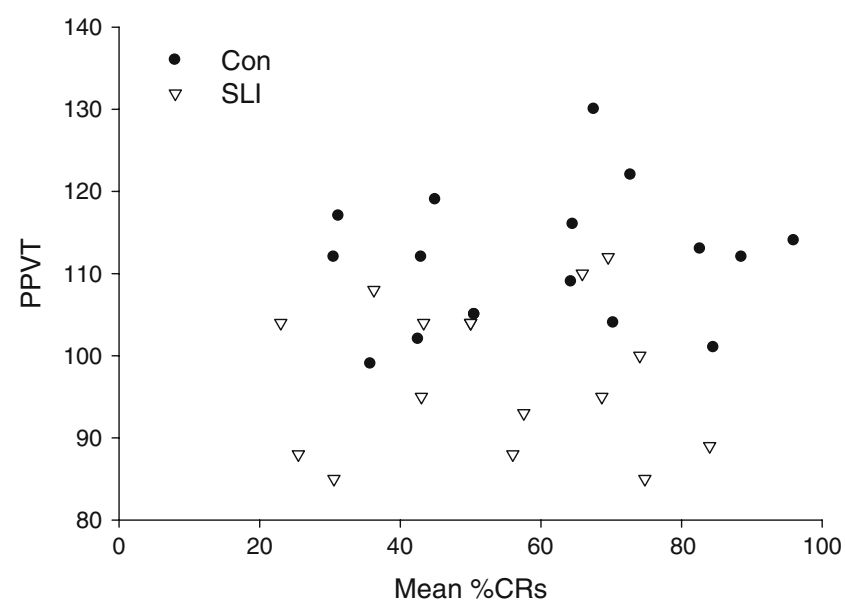

Fig. 2 Correlation of PPVT scores vs average percent CRs for controls and SLI participants. There was no significant correlation 
language, or reading impairment. The results suggest that the cerebellar circuitry involved in delay eyeblink conditioning, using the fundamental single-cue delay EBS procedure, is not affected in this group of SLI participants without speech impairments but with persistent language and reading impairments. The advantage of the EBC procedure used here is that the underlying cerebellar neural pathways are well documented in particular detail. The outcomes do not support cerebellar impairment at the level of basic motor learning pathways as part of the pathogenesis of SLI, at least in the classic SLI profile of the children in this sample. In agreement with past studies (Sears et al. 1994) it was reported there were no age effects in the control groups on measures of conditioning-children ages 9-11 are as proficient as young adults. The findings here for children with SLI do not replicate the earlier report (Sears et al. 1994) of EBC differences in a group of children with autism versus control children. The methods of the two studies are very similar so presumably the lack of replication may be attributable to group differences. The findings also do not replicate the earlier report of EBC differences in a group of adolescents and young adults with dyslexia (Nicolson et al. 2002). The lack of replication in this case may be attributable to methodological differences or sampling differences. The methods of Nicolson et.al. involved a CS of $800 \mathrm{~ms}$, longer than usually used in EBC studies, which could influence responses; the number of learning trials was smaller than usual, with 42 paired tone-airpuff trials instead of the 100 trials used in this study; and the percentage of CR differences between groups were not reported. The sample of persons with dyslexia is also different from this study. The participants with dyslexia in Nicolson et. al scored in the normal or above normal levels on verbal tasks. It is quite possible that persons with reading impairments without language impairments are different from persons with reading and language impairments.

Overall, the results of this study suggest that children with SLI and controls both learned the response and at a similar rate. The groups did not differ in terms of other learning factors such as peak onset of the CR response or the CR and UR amplitudes. Overall, the findings suggest that the circuitry involving delay eyeblink conditioning, as measured in this procedure, including the cerebellum, is intact in children with SLI.

The cerebellum has also been implicated to be necessary for the learning of vocabulary. PET studies have shown an activation of the cerebellum during verbal short term memory tasks (Paulesu et al. 1993). This has also been verified with a case study of an individual with a cerebellar lesion (Silveri et al. 1998). Since eyeblink/cerebellar learning and vocabulary knowledge is sufficiently intact for children with SLI, this could explain why it does not predict individual differences in vocabulary outcomes (i.e. PPVT). This is consistent with other studies which have found no correlations with a variety of intelligence performance variables correlated with eyeblink conditioning (Cromwell et al. 1961; Ohlrich and Ross 1968). This study also replicated findings that young adults do not differ from adolescents on the level of eyeblink conditioning (Sears et al. 1994).

Importantly, this sample of children with SLI is without a history of speech impairments. Although reviews sometimes conflate SLI and impairments of speech production (Hill 2001), an epidemiologically ascertained sample of children yielded an estimated $2 \%$ of overlap of speech and language impairments; for the children with SLI, speech disorders were evident in approximately $5-8 \%$ of the children (Shriberg et al. 1999). The findings here are consistent with the possibility that cerebellar impairment could be implicated in motor speech components instead of the grammar components of children with SLI. At the same time, the study also suggests that learning at the level of the EBC, under cerebellar control, is not likely to be a source of the persistent grammatical deficits characteristic of SLI, given that the participants had grammatical deficits and unaffected EBC. Under this logic, the outcomes suggest that EBC-related cerebellar circuitry is not implicated in a possible cerebellar role in reading impairments, as hypothesized by Nicolson, et al. (Nicolson et al. 2001), given that this sample of children with SLI showed persistent low levels of performance on word identification tasks although there were no group differences on the EBC learning measures. The alternative possibility is that EBC impairments are present in some but not all groups diagnosed with dyslexia.

There are several limitations to the current study which must be considered. First, the delay eyeblink conditioning paradigm circuitry involves the entire cerebellum. It is possible that deficits could be present with specific regions of the cerebellum such as the anterior cortex. In this case, other variations of eyeblink conditioning, such as a long ISI, could be employed to probe anterior cerebellar cortex function. Second, young infants can demonstrate acquisition that is indistinguishable from adults in terms of asymptote of learning and timing of responding in a delay conditioning task (Herbert et al. 2003). Thus, a task involving increased demands on the cerebellum could provide more insight into the possible roles of the cerebellum in SLI. Finally, although the sample size might be considered overly small to detect group differences, it is unlikely to have been insufficient given the small between-group effect size of the primary variable of interest, acquisition phase \% CRs (i.e., main effect of group partial eta ${ }^{2}$ was .01). 
In conclusion, the results of the study indicate participants with SLI acquire a cerebellar-dependent task at a similar rate to controls. SLI participants also show no differences in timing and amplitude of the response. This would suggest that the cerebellum of participants with SLI without speech impairments (but with reading impairments) is not grossly impaired and thus the deficits witnessed with participants with SLI are likely to be localized to other areas on the brain.

Acknowledgements We thank Alyson Abel and Megan Blossom for assistance with data collection, Denise Perpich for assistance with data analyses, Doug Kieweg and Bruno Tagliaferri for assistance with instrumentation, and Jeff Admundson, Rich Vogel and Joseph Steinmetz for contribution to interpretive discussions. Most of all we express our appreciation to the children and adults who participated in the experimental procedures. This research was funded by the National Institutes of Health P30DC005803, R01DC001803, and R01DC005226 to Mabel Rice, Principal Investigator, as well as by the University of Kansas Intellectual and Developmental Disabilities Research Center P30HD002528 to John Colombo.

\section{References}

Akshoomoff NA, Courchesne E. A new role for the cerebellum in cognitive operations. Behav Neurosci. 1992;106:731-8.

Alarcón M, Abrahams BS, Stone JL, Duvall JA, Perederiy JV, Bomar $\mathrm{JM}$, et al. Linkage, association, and gene-expression analyses identify CNTNAP2 as an autism-susceptibility gene. Am J Hum Genet. 2008;82:150-9.

Bishop DVM. Motor immaturity and specific speech and language impairment: evidence for a common genetic basis. Am J Med Genet (Neuropsychiatric Genetics). 2002;114:56-63.

Blaxton TA, Zeffiro TA, Gabriele JDE, Bookheimer SY, Carrillo MC, Theodore WH, et al. Functional mapping of human learning: a positron emission tomography activation study of eyeblink conditioning. J Neurosci. 1996;16:4032-40.

Brown SM, Kieffaber PD, Carroll CA, Vohs JL, Tracy JA, Shekhar A, et al. Eyeblink conditioning deficits indicate timing and xcerebellar abnormalities in schizophrenia. Brain Cogn. 2005;58:94-108.

Burgemeister BB, Blum LH, Lorge I. The Columbia mental maturity scale. San Antonio: Psychological Corporation; 1972.

Catts HW. Language impairments and reading disabilities. In: Kent RD, editor. THE MIT Encyclopedia of communication disorders. Cambridge: MIT; 2004. p. 329-31.

Christian KM, Thompson RF. Neural substrates of eyeblink conditioning: acquisition and retention. Learn Mem. 2003;10:427-55.

Coffin JM et al. Impaired cerebellar learning in children with prenatal alcohol exposure: a comparative study of eyeblink conditioning in children with ADHD and dyslexia. Cortex. 2005;41:389-98.

Cohen J. Statistical power analysis and research results. Am Educ Res J. 1973;10:225-9.

Conti-Ramsden G, Simkin Z, Botting N. The prevalence of autistic spectrum disorders in adolescents with a history of specific language impairment (SLI). J Child Psychol Psychiatry. 2006;47 (6):621-8.

Cromwell R, Palk B, Folshee J. Studies in activity level: V. The relationships among eyelid conditioning, intelligence, activity level, and age. Am J Ment Defic. 1961;65:744-8.

Daum I, Schugens MM, Ackermann H, Lutzenberger W, Dichgans J, Birbaumer N. Classical conditioning after cerebellar lesions in humans. Behav Neurosci. 1993;107:748-56.
Diamond A. Close interrelation of motor development and cognitive development and of the cerebellum and prefrontal cortex. Child Dev. 2000;71:44-56.

Dimitrova A, Weber J, Redies C, Kindsvater K, Maschke M, Kolb FP, et al. MRI atlas of the human cerebellar nuclei. Neuroimage. 2002;17:240-55.

Gerwig M, Hajjar K, Dimitrova A, Maschke M, Kolb FP, Frings M, et al. Timing of conditioned eyeblink responses is impaired in cerebellar patients. J Neurosci. 2005;25:3919-31.

Gerwig M, Kolb FP, Timmann D. The involvement in the human cerebellum in eyeblink conditioning. Cerebellum. 2007;6:38-57.

Goldman R, Fristoe M. The Goldman-Fristoe test of articulation. Circle Pines: American Guidance Service; 1986.

Herbert J, Eckerman C, Stanton M. The Ontogeny of human learning in delay, long-delay, and trace eyeblink conditioning. Behav Neurosci. 2003;117:1196-210.

Hill EL. Non-specific nature of specific language impairment: a review of the literature with regard to concomitant motor impairments. Int J Lang Commun Disord. 2001;36:149-71.

Justus T. The cerebellum and English grammatical morphology: evidence from production, comprehension, and grammaticality judgments. J Cogn Neurosci. 2004;16:1115-30.

Kim JJ, Thompson RF. Cerebellar circuits and synaptic mechanisms involved in classical eyeblink conditioning. Trends Neurosci. 1997;20:177-81.

King D, Tracy J. Datamunch: a Matlab m-file collection for the analysis of trial-based spike and behavioral data http://www. nov1.indiana.edu/Bdmunch/; 1999.

Kjelgaard M, Tager-Flusberg H. An investigation of language profiles in autism: implications for genetic subgroups. Lang Cognit Processes. 2001;16:287-308.

Lavond DG, Steinmetz JE. Acquisition of classical conditioning without cerebellar cortex. Behav Brain Res. 1989;33:113-64.

Leiner HC, Leiner AL, Dow RS. The human cerebro-cerebellar system: its computing, cognitive and language skills. Behav Brain Res. 1991;44:113-28.

Leonard LB, Weismer SE, Miller CA, Francis DJ, Tomblin JB, Kail RV. Speed of processing, working memory, and language impairment in children. J Speech Lang Hear Res. 2007;50:408-28.

Lindgren KA, Folstein SE, Tomblin JB, Tager-Flusberg H. Language and reading abilities of children with autism spectrum disorders and specific language impairment and their first-degree relatives.. Autism. Research. 2009;2:22-38.

Logan C. Cerebellar cortical involvement in excitatory and inhibitory classical conditioning. Palo Alto: Stanford University; 1991.

Logan CG, Grafton ST. Functional anatomy of human eyeblink conditioning determined with regional cerebral glucose metabolism and positron-emission tomography. Proc Nat Acad Sci. 1995;92:7500-4.

Lum JAG, Conti-Ramsden G, Lindell AK. The attentional blink reveals sluggish attentional shifting in adolescents with specific language impairment. Brain Cogn. 2007;63:287-95.

Lye RH, O' Boyle DJ, Ramsden RT, Schady W. Effects of a unilateral cerebellar lesion on the acquisition of eye-blink conditioning in man. J Physiol (Lond). 1988;403:58.

McCormick DA, Thompson RF. Cerebellem: esssential involvement in the classically conditioned eyelid response. Science. 1984;223:296-9.

McGlinchey-Berroth R, Cermak LS, Carrillo MA, Armfield S, Gabrieli JDE, Disterhoft JF. Impaired delay eyeblink conditioning in amnesic Korsakof's patients and recovered alcoholics. Alcohol Clin Exp Res. 1995;19(5):1127.

Nicolson RL, Fawcett AJ. Procedural learning difficulties: reuniting the developmental disorders? Trends Neurosci. 2007;30:135-41.

Nicolson RL, Fawcett AJ, Dean P. Developmental dyslexia: the cerebellar deficit hypothesis. Trends Neurosci. 2001;24:508-11. 
Nicolson RL, Daum I, Schugens MM, Fawcett AJ, Schulz A. Eyeblink conditioning indicates cerebellar abnormality in dyslexia. Exp Brain Res. 2002;143:42-50.

Ohlrich E, Ross L. Acquisition and differential conditioning of the eyelid response in normal and retarded children. J Exp Child Psychol. 1968;6:181-93.

Paulesu E, Frith CD, Frackowiak RSJ. The neural correlates of the verbal component of working memory. Nature. 1993;362:342-5.

Perrett SP, Ruiz BP, Mauk MD. Cerebellar cortex lesions disrupt learning-dependent timing of conditioned eyelid responses. J Neurosci. 1993;13:1708-18.

Ramnani N, Toni I, Josephs O, Ashburner J, Passingham R. Learningand expectation-related changes in the human brain during motor learning. J Neurophysiol. 2000;84:3026-35.

Rapoport M, Van Reekum R, Mayberg H. The role of the cerebellum in cognition and behavior: a selective review. J Neuropsychiatry Clin Neurosci. 2000;12:193-8.

Rice ML. Grammatical symptoms of specific language impairment. In: Bishop DVM, Leonard LB, editors. Speech and language impairments in children: Causes, characteristics, intervention and outcome. East Sussex: Psychology Press Ltd; 2000. p. 17-34.

Rice ML, Wexler K. Toward tense as a clinical marker of specific language impairment in English-speaking children. J Speech Hear Res. 1996;39:1239-57.

Rice ML, Wexler K. Rice/Wexler test of early grammatical impairment. San Antonio: The Psychological Corporation; 2001.

Rice ML, Wexler K, Cleave PL. Specific language impairment as a period of extended optional infinitive. J Speech Lang Hear Res. 1995;38:850-63.

Rice ML, Hoffman L, Wexler K. Judgments of omitted BE and DO in questions as extended finiteness clinical markers of specific language impairment (SLI) to 15 years: a study of growth and asymptote. J Speech Lang Hear Res. 2009a;52:1417-33.

Rice ML, Smith SD, Gayán J. Convergent genetic linkage and associations to language, speech and reading measures in families of probands with Specific Language Impairment. J Neurodev Disord. 2009b. doi:10.1007/s11689-009-9031-x.

Roberts JA, Rice ML, Tager-Flusberg H. Tense marking in children with SLI: further evidence for overlap between autism and SLI. Appl Psycholinguist. 2004;25:429-48.

Rogers RF, Britton GB, Steinmetz JE. Learning-related interpositus activity is conserved across species as studied during eyeblink conditioning in the rat. Brain Res. 2001;905:171-7.

Schugens MM, Daum I. Long-term retention of classical eyeblink conditioning in amnesia. NeuroReport. 1999;10:149-52.

Sears LL, Finn PR, Steinmetz JE. Abnormal classical eye-blink conditioning in autism. J Autism Dev Disord. 1994;24:737-51.

Shriberg LD, Tomblin JB, McSweeny JL. Prevalence of speech delay in 6-year-old children and comorbidity with language impairment. J Speech Lang Hear Res. 1999;42:1461-81.
Silveri MC, Di Betta AM, Filippini V, Leggio MG, Molinari M. Verbal short-term store-rehearsal system and the cerebellum. Brain. 1998;121:2175-87.

Skosnik PD, Edwards CR, O’Donnell BF, Steffen A, Steinmetz JE, Hetrick WP. Cannabis use disrupts eyeblink conditioning: evidence for cannabinoid modulation of cerebellar-dependent learning. Neuropsychopharmacology. 2008;33:1432-40.

Solomon PR, Pomerleau D, Bennett L, James J, Morse DL. Acquisition of the classically conditioned eyeblink response in humans over the life span. Psychol Aging. 1989;4:34-41.

Steinmetz JE. Brain substrates of classical eyeblink conditioning: a highly localized but also distributed system. Behav Brain Res. 2000;110:13-24.

Tager-Flusberg H, Cooper J. Present and future possibilities for defining a phenotype for specific language impairment. J Speech Lang Hear Res. 1999;42:1275-8.

Tomblin JB, Records NL, Buckwalter P, Zhang X, Smith E, O'Brien M. The prevalence of specific language impairment in kindergarten children. J Speech Hear Res. 1997;40:124560.

Topka H, Valls-Solé J, Massaquoi SG, Hallett M. Deficit in classical conditioning in patients with cerebellar degeneration. Brain. 1993;116:961-9.

Vernes SC, Newbury DF, Abrahams BS, Winchester L, Nicod J, Groszer $\mathrm{M}$, et al. A functional genetic link between distinct developmental language disorders. N Engl J Med. 2008;359 (22):2337-45.

Wechsler D. Wechsler intelligence scale for children. 3rd ed. San Antonio: The Psychological Corporation; 1991.

Woodcock RW. Expressive vocabulary test. Circle Pines: American Guidance Service; 1987.

Woodruff-Pak DS, Thompson RF. Classical conditioning of the eyeblink response in the delay paradigm in adults aged 18 83 years. Psychol Aging. 1988;3:219-29.

Woodruff-Pak DS, Papka M, Ivry RB. Cerebellar involvement in eyeblink classical conditioning in humans. NeuropsychologyNew York. 1996;10:443-58.

Woodruff-Pak DS, Jaeger ME, Gorman C, Wesnes KA. Relationship among age, conditioned stimulus-unconditioned stimulus interval, and neuropsychological test performance. Neuropsychology. 1999;13:90-102.

Yamaguchi S, Tsuchiya H, Kobayashi S. Visuo-spatial attention shift and motor responses in cerebellar disorders. J Cogn Neurosci. 1998;10:95-107.

Zelaznik HN, Goffman L. Generalized motor abilities and timing behavior in children with specific language impairment. J Speech Lang Hear Res. 2010;53:383-93.

Zubrick SR, Taylor CL, Rice ML, Slegers D. Late language emergence at 24 months: an epidemiological study of prevalence and covariates. J Speech Lang Hear Res. 2007;50:1562-92. 